\title{
Consistency of High Performance Liquid Chromatography, Electrochemiluminescence and Mass Spectrometry Methods for Vitamin D Measurement
}

\author{
Hülya Çiçek $^{\mathbf{1}}$, Ümmü Gülsüm Çiftçi Aksoy ${ }^{\mathbf{1}}$ and Seval Kul ${ }^{\mathbf{2}}$ \\ ${ }^{1}$ Department of Medical Biochemistry, Faculty of Medicine, University of Gaziantep, Turkey \\ ${ }^{2}$ Department of Biostatistics, Faculty of Medicine, University of Gaziantep, Turkey \\ *Corresponding Author: Hülya ÇIÇEK, Professor, Gaziantep University, School of Medicine, Medical Biochemistry Department, \\ Şahinbey-Gaziantep, Turkey.
}

Received: May 20, 2019; Published: July 04, 2019

DOI: $10.31080 /$ ASMS.2019.03.0338

\begin{abstract}
Purpose: To validate High Performance Liquid Chromatography and Electrochemiluminescence methods as well- standardized assay: Mass Spectrometry and measure variations among methods for testing vitamin D.

Methods: The study was conducted with a total of 57 volunteers who admitted to the Central Laboratory Blood Collection Unit of Gaziantep University. 25(OH) vitamin D levels were measured by High Performance Liquid Chromatography (HPLC), Liquid Chromatography-Mass Spectrometry (LC-MS/MS) and Electrochemiluminescence (ECL) methods in serum samples. Intra-observer agreement between studied methods was evaluated by Intra-class correlation coefficient (ICC) and Pearson correlation coefficient (r). Furthermore, Deming regression which takes into account measurement errors for both methods was also performed to compare methods. Results: The highest ICC was found for HPLC versus LC-MS/MS (ICC=0.961, 95\% CI 0.935-0.977, p=0.001). Also, Pearson correlation coefficient was greater than 0.80 for all pairwise comparisons. According to the results of Deming regression, 3 methods were correlated with each other with slopes ranging from 0.91 to 1.07 . However, the highest ICC and linear correlation were observed between LC-MS/MS and HPLC methods.

Conclusion: The highest ICC and correlation coefficient were obtained between LC-MS/MS and HPLC methods. A smaller variation was found between HPLC and LC-MS/MS methods, particularly at low vitamin D levels. Our study showed that in addition to the reference test, both ECL and HPLC (and preferably HPLC) are reliable methods to estimate vitamin D levels by measuring 25(OH) D levels in human serum. The highest ICC and correlation coefficient were obtained between LC-MS/MS and HPLC methods. A smaller variation was found between HPLC and LC-MS/MS methods, particularly at low vitamin D levels. Our study showed that in addition to the reference test, both ECL and HPLC (and preferably HPLC) are reliable methods to estimate vitamin D levels by measuring 25(OH) D levels in human serum.

Keywords: Method Comparison; Vitamin D; HPLC; ECL; LC-MS/MS
\end{abstract}

\section{Abbreviations}

25(OH) D: 25(OH) vitamin D; HPLC: High Performance Liquid Chromatography; LC-MS/MS: Liquid Chromatography-Mass Spectrometry; ECL: Electrochemiluminescence; ICC: Intra-Class Correlation Coefficient; CV: Coefficient of Variation; CLIA: Chemiluminescence Immunoassay; RIA: Radioimmunoassay; DBP: Vitamin D Binding Protein.

\section{Introduction}

Vitamins are essential compounds for human organism which act as a cofactor in enzymatic reactions [1-3]. Vitamin D is a fatsoluble vitamin that can be classified as a hormone by having a chemical structure that is similar to steroid hormones and by being synthesized in a tissue and released into the circulatory system 
to exert its effects in the target tissue [2]. Serum levels of 25-hydroxyvitamin D (25(OH)D) are considered the best biomarker of vitamin D nutritional status [4]. Vitamin D has become the focus of many recent studies. The U.S. Institute of Medicine considers that a serum 25-hydroxyvitamin D (250HD) concentration $>20 \mathrm{ng} / \mathrm{mL}$ corresponds to optimal vitamin D status in the general population [5]. Vitamin D deficiency has become a significant problem which is now considered as pandemic [6]. 250HD concentrations of $<12$ and $<20 \mathrm{ng} / \mathrm{mL}$ were found in $9.9 \%$ and $34.6 \%$ of healthy French adults respectively [7]. Vitamin D deficiency has been implicated in many skeletal and non-skeletal health problems. The U.S. Institute of Medicine claimed a key role of vitamin D in skeletal health [5]. It is widely known that vitamin D deficiency causes rickets in children and it may lead to and worsen osteopenia, osteoporosis, and fractures in adults [6]. Furthermore it was found that a high concentration of plasma 25(OH)D and vitamin D are associated with a reduced risk of premenopausal breast cancer, which is one of the most prevalent cancer all over the world [8,9]. In addition to breast cancer, low vitamin D levels were found to be associated with colorectal, prostate, ovarian cancer, autoimmune diseases, Type II diabetes and cardiovascular diseases [4]. Levels of 25-hydroxyvitamin D (25-OH D) during late pregnancy have been linked to type 1 diabetes risk in the offspring. One of the most recent studies showed that lower third trimester concentrations of vitamin D binding protein (DBP) and $25(\mathrm{OH}) \mathrm{D}$ tended to be associated with higher risk of type 1 diabetes in the children [10].

Accurate measurement of Vitamin D level is critical to diagnose, prevent and treat Vitamin D deficiency,. 25(OH)D was found to be stable at room temperature for at least $72 \mathrm{~h}$ in either serum or whole blood [11]. The stability of serum 25(OH)D was confirmed by DiaSorin RIA for up to four freeze-thaw cycles and exposure to UV light or freeze-thaw cycles (up to eleven times) [13,14].

Earlier assays applied the competitive protein binding format using the DBP as the binder; then, in 1985, a radioimmunoassay (RIA) was developed for 25(OH)D. However, both the DBP and the RIA assays typically overestimated $25(\mathrm{OH}) \mathrm{D}$ levels by approximately $10-20 \%[14,15]$. Immunoassay methods are not sufficiently reliable for measuring the amount of 25(OH)D because of crossreactions. Later, HPLC was considered to be the gold standard but was a very cumbersome assay and thus, was not routinely used by reference laboratories for clinical samples [16].
Also, the advances in liquid chromatography tandem mass spectrometry (LC-MS) led to its use for direct measurement of $25(\mathrm{OH})$ D in human serum and it replaced HPLC as the new gold standard for quantitative measurement of different types of vitamin D molecules separately. Today, laboratories are using chemiluminescence immunoassay (CLIA), radioimmunoassay (RIA), high performance liquid chromatography (HPLC) and liquid chromatography-tandem mass spectrometry (LC-MS/MS) methods for determination of serum 25(OH)D levels. To minimize the methodological differences between assays in measuring vitamin $\mathrm{D}$, a reference material is needed to standardize assays and eventually harmonize patient results. Currently, researchers are still seeking an internationally recognized reference standard to be used for assessment of vitamin D status [17].

The aim of this study was to validate High Performance Liquid Chromatography (HPLC), Liquid Chromatography-Mass Spectrometry (LC-MS/MS) and Electrochemiluminescence (ECL) methods as well-standardized assays which are urgently needed in clinical laboratories and eliminate variations among methods for testing vitamin D for purposes of standardization and harmonization that.

\section{Materials and Methods}

The present study was approved by Gaziantep University Ethics Committee (Decision No. 24.02.2014/73 of 24.02.2014) and conducted at the Department of Medical Biochemistry of Gaziantep University Medical Faculty in accordance with the principles set forth in the Declaration of Helsinki. The study was supported by Gaziantep University Commission for Scientific Research Projects (TF.15.05).

The study was conducted with a total of 57 volunteers who admitted to the Central Laboratory Blood Collection Unit of Gaziantep University for measurement of 25(OH)D. Written informed consent was obtained from patients and/or their relatives for participation in the study.

Blood sampling was performed at the blood collection unit of Gaziantep University Medical Faculty Hospital. $10 \mathrm{cc}$ of blood was collected from the antecubital vein into plain tubes while the subjects were seated. Blood samples were divided into 3 portions of 1-1,5 cc and transferred into eppendorf tubes and labelled; then, they were stored at $-80^{\circ} \mathrm{C}$ until the time of analysis. Hemolyzed or lypemic sera were excluded from the study. 
Consistency of High Performance Liquid Chromatography, Electrochemiluminescence and Mass Spectrometry Methods for Vitamin D Measurement

Age and gender data were retrieved from patient files. $25(\mathrm{OH})$ vitamin D levels were measured by High Performance Liquid Chromatography (HPLC), Liquid Chromatography-Mass Spectrometry (LC-MS/MS) and Electrochemiluminescence (ECL) methods in serum samples. Chemiluminescent analyses were performed by the Central Laboratory of Gaziantep University Medical Faculty Hospital, whereas HPLC and MS assays were conducted by an outsourced facility.

\section{Exclusion criteria}

Patients using vitamin D preparations were excluded from the study.

\section{Measurement of Parameters}

Vitamin D measurement using HPLC method

In this method, $25(\mathrm{OH})$ vitamin D levels in blood samples were obtained by a Shimadzu Prominence UFLC System (Shimadzu, Kyoto, Japan) device.

Patient blood samples were subjected to pretreatment for determination of $25(\mathrm{OH})$ vitamin D levels by high performance liquid chromatography. Therefore, precipitation step was combined with extraction. Shim-pack XR-ODS (100 mm x $3.0 \mathrm{~mm}$ ) column was used for the assessment. Mobile phase Acetonitrile/Ethanol=90/10 (v/v), flow rate was $1.0 \mathrm{~mL} / \mathrm{min}$ and column temperature was $25^{\circ} \mathrm{C}$. Detections were recorded absorbance at $265 \mathrm{~nm}$ with conventional flow cell and each injection volume was $8 \mu \mathrm{L}$. The analysis was conducted according to the procedure described in the kit insert. The standard mix was prepared using ethanol to bring each of the vitamin D concentrations to $0.1 \mathrm{mg} / \mathrm{L}$.

Following centrifugation of the sample, upper layer was injected into the HPLC system. The HPLC separation works with an isocratic method with a reversed phase column. Chromatograms were detected by an UV detector. Results were quantified by the delivered serum calibrator and calculated by the internal standard method by integration of the peak areas or heights.

\section{Vitamin D measurement using LC-MS method}

Shimadzu Triple Quadrupole 8040 (Japan) device with APCI source in positive mode was used to quantify $25(\mathrm{OH})$ vitamin D in patient blood samples by LC-MS method using Perkin Elmer kit. The assay was conducted according to the procedure described in the device insert.
The analysis were performed in following these conditions;

Flow: $0.5 \mathrm{~mL} / \mathrm{min}$, run: $6 \mathrm{~min}$, injection volume: $35 \mu \mathrm{L}$, mobile phase A: water, mobile phase B: methanol, gradients were $(0 \mathrm{~min} B$ 50\%,3 min B 100\%, 4 min B 100\%, 4.1 min B 50\%) respectively. DL $150^{\circ} \mathrm{C}$, nebulizing gas $2.5 \mathrm{~L} / \mathrm{min}$, heat block $250{ }^{\circ} \mathrm{C}$, drying gas $5 \mathrm{~L} /$ min and column polaris $3 \mathrm{C} 1850 \mathrm{~mm} \times 2 \mathrm{~mm} 3 \mu \mathrm{m}$.

For determination of $25(\mathrm{OH})$ vitamin $\mathrm{D}$, first a precipitation procedure was performed to remove high molecular weight substances, followed by centrifugation. Resulting supernatant was injected into the HPLC system. For HPLC separation, an isocratic method was employed using a reversed phase column at $20-25^{\circ} \mathrm{C}$. Chromatograms were detected by a MS/MS detector (AB-Sciex API 4000). Results were quantified by the delivered serum calibrator and calculated by the internal standard method by integration of the peak areas or heights.

Measurement of vitamin D by electrochemiluminescence method

$25(\mathrm{OH})$ vitamin D levels in patient blood samples were determined by a Roche/Hitachi Cobas 800 (Germany) device which is often preferred by diagnostic laboratories. The principle of this method is competitive protein binding assay.

The vitamin D total assay employs VDBP to capture both 25-hydroxyvitamin D3 and D2. This assay is used for quantitative determination of total vitamin D $(25-\mathrm{OH})$ in human serum and plasma, as an aid in the assessment of vitamin D sufficiency. First, the sample is incubated for 9 minutes with a pretreatment reagent. As a result, the natural VDBP in the sample is denatured to release the bound $25(\mathrm{OH})$ vitamin D. Second, the sample is further incubated with a recombinant ruthenium-labeled VDBP to form a complex of $25(\mathrm{OH})$ vitamin D and the ruthenylated-VDBP. Third, with the addition of a biotinylated $25(\mathrm{OH})$ vitamin $\mathrm{D}$ a complex consisting of the ruthenium-labeled VDBP and the biotinylated 25(OH) vitamin $\mathrm{D}$ is formed. The entire complex becomes bound to the solid phase (by the interaction of biotin and streptavidin-coated microparticles which are captured on the surface of the elecrode). Unbound substances are removed. Applying voltage to the electrode induces chemiluminescent emission which is measured by a photomultiplier. Results are determined by an instrument-specific calibration curve which is generated by 2-point calibration and a calibration master curve provided by the reagent barcode. 
Detection limit of the test is $3.00 \mathrm{ng} / \mathrm{mL}$ and functional sensitivity is $4.01 \mathrm{ng} / \mathrm{mL}$. The measuring range of the assay is between 3.00 and $70.0 \mathrm{ng} / \mathrm{mL}$. Intra-assay and inter-assay coefficient of variations (CVs) are 3.9-5.4\% and 3.9-18.5\%, respectively.

\section{Statistical method}

Intra-observer agreement between studied methods was evaluated by Intra-class correlation coefficient (ICC) and Pearson correlation coefficient (r). Excellent consistency was considered when both ICC and $r$ were greater than 0.80. Furthermore, Deming regression which takes into account measurement errors for both methods was also performed to compare methods. All statistical analyses were performed by SPSS for Windows, version 22.0 (IBM Corp. Released 2013) and Med Calc Statistical Software version 15.11.0 (MedCalc Software bvba, Ostend, Belgium). All coefficients were considered significant when the $95 \%$ confidence interval did not include " 0 ".

\section{Power analysis}

It was calculated that a minimum sample size of 30 in each group would be needed in order to demonstrate a moderate significant correlation $(r=0.50)$ between the methods $(\alpha=0.05$, $1-\beta=0.80)$.

\section{Results and Discussion}

R A total of 57 subjects with a mean ( \pm SD) age of $46.5 \pm 17.6$ years participated in the study including 13 males $(22.8 \%)$ and 44 females (77.2\%) (Table 1). 25(OH) vitamin D levels were measured in patient serum samples using ECL, HPLC and LC-MS/MS.

25(OH) Vitamin D levels were $19.73 \pm 11.87 \mathrm{ng} / \mathrm{mL}$ as determined by ECL, $18.87 \pm 12.47 \mathrm{ng} / \mathrm{mL}$ by HPLC and $18.40 \pm 10.90$ $\mathrm{ng} / \mathrm{mL}$ by LC-MS/MS (Table 1, Figure 1 ).

\begin{tabular}{|l|l|l|l|l|}
\hline \multicolumn{1}{|c|}{ Variables } & N & \multicolumn{1}{|c|}{ Min-Max } & \multicolumn{1}{c|}{$\begin{array}{c}\text { Mean } \pm \text { Std. } \\
\text { Dev. }\end{array}$} & CV* $^{*}$ \\
\hline Age & 57 & $18.0-65.00$ & $46.5 \pm 17.6$ & - \\
\hline $\begin{array}{l}\text { Electrochemilumi- } \\
\text { nescence (ng/mL) }\end{array}$ & 57 & $5.93-61.64$ & $19.73 \pm 11.87$ & 0.60 \\
\hline HPLC (ng/mL) & 57 & $3.66-68.28$ & $18.87 \pm 12.47$ & 0.66 \\
\hline $\begin{array}{l}\text { Mass Spectrometry } \\
\text { (ng/mL) }\end{array}$ & 57 & $5.00-58.40$ & $18.40 \pm 10.90$ & $\begin{array}{l}0 \\
69\end{array}$ \\
\hline
\end{tabular}

Table 1: Descriptive statistics for numerical variables. * Coefficient of variation

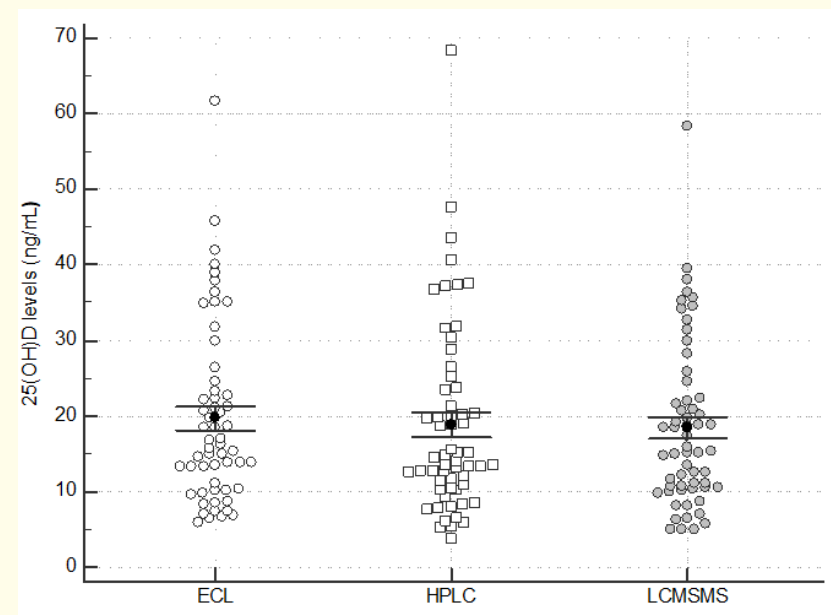

Figure 1: Comparison of $25(\mathrm{OH})$ D levels measured by three different methods.

Coefficient of variations for three different methods were 0.60 , 0.66 and 0.69 , respectively. Intra-class correlation coefficients (ICC) showed perfect agreement between methods. ICCs and $95 \%$ confidence intervals are provided in Table 2.

\begin{tabular}{|l|c|c|}
\hline \multicolumn{1}{|c|}{ Pairs } & ICC (95\% CI) & r (95\%CI) \\
\hline ECL vs LC-MS/MS & $0.924(0.871-0.955)$ & $0.863[0.770-0.917]$ \\
\hline HPLC vs LC-MS/MS & $0.961(0.935-0.977)$ & $0.934[0.891-0.960]$ \\
\hline ECL vs HPLC & $0.911(0.848-0.947)$ & $0.837[0.732-0.902]$ \\
\hline
\end{tabular}

Table 2: Correlation coefficients to compare methods.

ICC: Intraclass correlation coeficient; r: correlation coefficient.

The highest ICC was found for HPLC versus LC-MS/MS (ICC $=0.961,95 \%$ CI 0.935-0.977, p=0.001). Also, Pearson correlation coefficient was greater than 0.80 for all pairwise comparisons.

LC-MS/MS method was considered as gold standard and Deming regression was used to evaluate estimation performance of ECL and HPCL methods in comparison to LC-MS/MS method with regard to vitamin D levels. Results are given in Table 3.

According to the results of Deming regression, 3 methods were correlated with each other with slopes ranging from 0.91 to 1.07 . However, the highest ICC and linear correlation were observed between LC-MS/MS and HPLC methods (Table 2). In HPLC method not only the slope, but also the intercept were statistically signifi- 
Consistency of High Performance Liquid Chromatography, Electrochemiluminescence and Mass Spectrometry Methods for Vitamin D Measurement

\begin{tabular}{|l|c|c|c|}
\hline \multicolumn{1}{|c|}{ Pairs } & Intercept [95\% CI] & Slope [95\% CI] & Regression Equation \\
\hline ECL to estimate LC-MS/MS & $0.10[-5.14-5.33]$ & $0.91[0.58-1.22]$ & $\mathrm{Y}=0.91^{*} \mathrm{X}$ \\
\hline HPLC to estimate LC-MS/MS & $1.90[0.22-3.58]$ & $0.87[0.76-0.98]$ & $\mathrm{Y}=1.90+0,87^{*} \mathrm{X}$ \\
\hline ECL to estimate HPLC & $-2.43[-9.34-4.47]$ & $1.07[0.65-1.48]$ & $\mathrm{Y}=1.07^{*} \mathrm{X}$ \\
\hline
\end{tabular}

Table 3: Results of Deming regression.

cant. In the absence of reference method, HPLC was considered as reference test. Figures 2 and 3 shows graphical representation of regression analysis data for ECL and HPLC respectively. In these figures, black solid lines indicate Deming regression, and black dashed lines show $\mathrm{x}=\mathrm{y}$ (reference line).

In Figure 2, regression line deviates more from the reference line compared to Figure 3. Both methods showed the same performance in estimating 25(OH) D levels lower than $20 \mathrm{ng} / \mathrm{ml}$ (Figure 3 ) but for higher values there was a deviation between reference and regression lines.

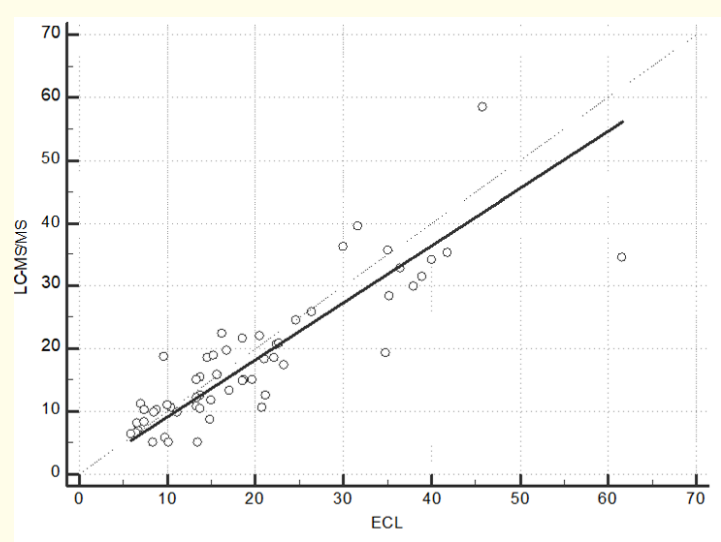

Figure 2: Deming regression graph to show 25(OH) D levels prediction performance of ECL versus LC-MS/MS.

Comparison of HPLC and ECL methods were given in (Figure 4). HPLC and ECL methods showed different performance in estimating 25(OH) D levels lower than $20 \mathrm{ng} / \mathrm{ml}$ but for higher values there was a no deviation between reference and regression lines.

The present study aimed to demonstrate consistency of 3 different methods used for assessment of Vitamin D levels. Fifty-seven randomly selected volunteers from 18 to 67 years of age were included in the study. Serum 25(OH)D levels were used to evaluate their vitamin D status. Three different statistical methods were used to examine the consistency of methods.

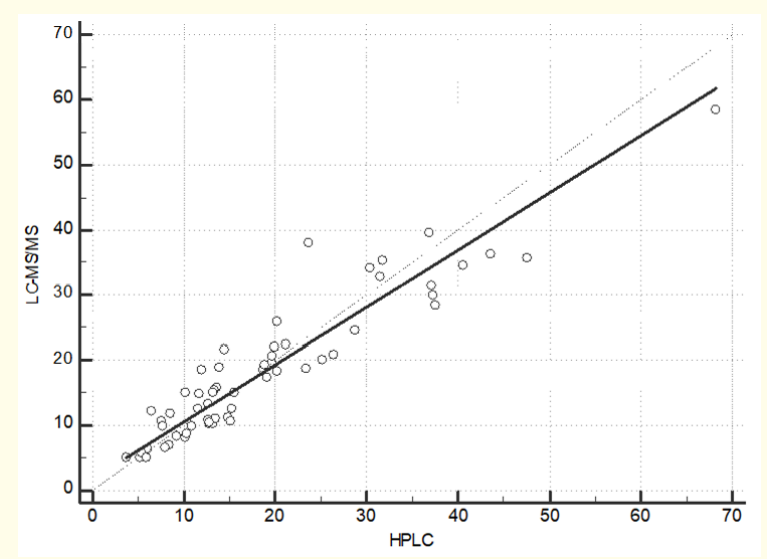

Figure 3: Deming regression graph to show 25(OH) D levels prediction performance of HPLC versus LC-MS/MS.

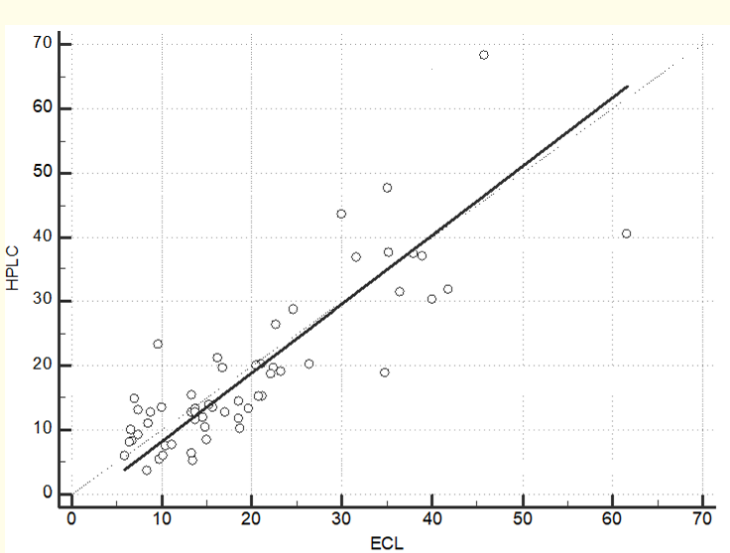

Figure 4: Deming regression graph to show 25(OH) D levels prediction performance of ECL versus HPLC.

For many years, measurement of $25(\mathrm{OH})$ vitamin D metabolites has gained much importance for elucidating various physiological and pathological events in the body. A method for $25(\mathrm{OH})$ vitamin D measurement was first reported in 1971 which is the competitive binding assay where vitamin D binding protein is used as the binder [17]. In this method, vitamin D binding protein binds to 
Consistency of High Performance Liquid Chromatography, Electrochemiluminescence and Mass Spectrometry Methods for Vitamin D Measurement

both $25(\mathrm{OH}) \mathrm{D}_{2}$ and $25(\mathrm{OH}) \mathrm{D}_{3}$ equally. Although this may seem like an advantage, the assay detects other polar vitamin D metabolites such as 24,25(OH)2D, 25,26(OH)2D and 23-lactone during measurement which limits its use in practice. An additional disadvantage of the method is the requirement for a long, 10 day incubation time. However, incubation times were reduced to 1 hour by developing a competitive protein binding assay that utilizes silicic acid chromatography $[16,18,19]$. In 1977, a specific HPLC method was developed in which the 25(OH)D fraction was applied to HPLC, and the UV absorption of $25(\mathrm{OH}) \mathrm{D}$ was used to measure its concentration [16]. The major advantage of this method is the ability to remove lipids and vitamin D metabolites that interfere with the assay, thereby allowing for measurement of $25(\mathrm{OH}) \mathrm{D}_{2}$ and $25(\mathrm{OH}) \mathrm{D}_{3}$. Nevertheless, this method requires expertise and suitable equipment [18,19]. In 1985, Radioimmumoassay (RIA) method was developed which does not require sample purification. RIA offers convenience of use and delivers results that are comparable with those of HPLC. As with competitive protein binding assay, RIA for $25(\mathrm{OH}) \mathrm{D}$ recognizes 25(OH)D2 and 25(OH)D3 to the same extent, and it can also detect other polar vitamin D metabolites. Thus, RIA assays typically overestimate $25(\mathrm{OH})$ vitamin D levels by approximately $10-20 \%[18-20]$.

As with competitive protein binding and RIA assays, the enzyme-linked immunosorbent assay (ELISA) method, detects other polar vitamin D metabolites as well as $25(\mathrm{OH}) \mathrm{D}_{2}$ and $25(\mathrm{OH}) \mathrm{D}_{3}$. Chemiluminescence method is equally specific for both $25(\mathrm{OH}) \mathrm{D}_{2}$ and $25(\mathrm{OH}) \mathrm{D}_{3}$ but requires expensive instrumentation [18]. Liquid Chromatography Tandem Mass Spectrometry (LC-MS/MS) quantitatively measures both $25(\mathrm{OH}) \mathrm{D}_{2}$ and $25(\mathrm{OH}) \mathrm{D}_{3}$. Also, it is considered as the reference method for vitamin D measurement due to its high sensitivity and specificity [21].

Recently, laboratory procedures employing immunological techniques and chromatographic methods for 25(OH)D measurement have been reviewed. Several investigators underscore the need for international standardization of 25(OH)D measurement and advocate the use of RIA for clinical evaluation [22]. Nevertheless, LC-MS/MS method which does not require radioactive materials delivers extremely accurate results. MS method is not commonly used in routine laboratories and mostly employed for research purposes. While GC-MS (Gas Chromatography Mass Spectrometry) method is utilized in studies for the analysis of vitamin D metabolites in plasma, it is not preferred due to its inherent complexity [23].
Recent studies reported that LC-MS/MS method has high sensitivity and specificity for many metabolites and suggested that it might be an alternative to immunoassay method, so offers a simple, linear and reproducible result. Thus, LC-MS/MS method has been suggested as the reference method for measurement of $25(\mathrm{OH})$ vitamin D [21] Gary L., et al. advocate that LC-MS/MS and HPLC methods should be preferred for accurate detection of vitamin D metabolites [24]. In one study, Alan H. Terry., et al. used chemiluminescence as the reference method and found a good agreement with LC-MS/MS [25]. Sahillioglu., et al. have also obtained consistent results for both methods [26]. In both studies, chemiluminescence method yielded higher values than LC-MS/MS and this is mainly because the chemiluminescence method measures the total 25(OH)D.

In Borai., et al. study, HPLC method was compared with two separate analyzers that use the chemiluminescence detection principle; it was shown that one of the analyzers produced results that correlated well with those of HPLC at higher concentrations while the other correlated with HPLC at lower concentrations. This underscores the importance of using assay-specific reference limits when interpreting the results for observed differences. In the present study, all three methods showed consistent results at low and high concentrations [27].

We used 25(OH)D (the major circulating form of vitamin D) to determine Vitamin D status which reflects the total amount of Vitamin $\mathrm{D}$ produced as a result of sun exposure and obtained by dietary intake $[28,29]$. Another popular way to measure Vitamin D level is to use $1,25(\mathrm{OH}) \mathrm{D}_{3}$ which is the biologically active form of vitamin $\mathrm{D}$ [28]. The half-life of circulating 1,25(OH)D is 4-6 hours. Circulating levels of $1,25(\mathrm{OH}) \mathrm{D}$ are a thousand fold less than 25(OH)D. When a patient has vitamin D deficiency, there is a increase in intestinal calcium absorption which lowers ionized calcium transiently. This signal is recognized by the calcium sensor in the parathyroid glands to increase the production and secretion of parathyroid hormone [30]. Parathyroid hormone controls calcium metabolism by increasing tubular reabsorption of calcium in the kidney, increasing mobilization of calcium from the skeleton and by increasing the renal production of 1,25(OH)D [31]. As a result, as a patient becomes vitamin D deficient and insufficient, the increase in PTH levels result in normal or elevated levels of 1,25(OH)D. This makes the $1,25(\mathrm{OH}) 2 \mathrm{D}$ assay useless as a correct measure of vitamin D status. 
Consistency of High Performance Liquid Chromatography, Electrochemiluminescence and Mass Spectrometry Methods for Vitamin D Measurement

There are some limitations of our study. Although participants reported no vitamin deficiency, 250HD concentrations $<12$ and $<20 \mathrm{ng} / \mathrm{mL}$ were found in $35.6 \%$ and $66.1 \%$. There may be 2 possible explanations for low vitamin D levels detected in our sample. First, this study was performed during winter em when ultraviolet rays are less intense. Furthermore, most of the female participants were wearing Islamic clothing which decreases sun exposure.

One of the limitations of the present study is the small sample size relative to some previous studies. However, our correlation coefficients were very high and our sample size was large enough to detect statistically significant correlations and regression coefficients. Still, one should exercise caution when using regression equations to estimate vitamin D levels measured by LC-MS/MS. In our sample, the difference between minimum and maximum vitamin D values were 53.40. This range may be wider in a real-world population. Also, it is known that with regard to simple regression, predictions are valid only within the range of values of variables used in the study [32]. Because of this feature of regression method, our regression equations cannot be extrapolated for every sample.

\section{Conclusion}

In conclusion, both correlation and regression analyses showed that 3 methods were highly correlated and results were similar. However, the highest ICC and correlation coefficient were obtained between LC-MS/MS and HPLC methods. A smaller variation was found between HPLC and LC-MS/MS methods, particularly at low vitamin D levels. Our study showed that in addition to the reference test, both ECL and HPLC (and preferably HPLC) are reliable methods to estimate vitamin D levels by measuring 25(OH) D levels in human serum.

\section{Acknowledgements}

The study was supported by Gaziantep University Commission for Scientific Research Projects (TF.15.05).

\section{Conflict of Interest}

The authors declare that they have no competing interests.

\section{Bibliography}

1. Holick MF. "Sunlight and vitamin D for bone health and prevention of autoimmune diseases, cancers, and cardiovascular disease". American Journal of Clinical Nutrition 80.6 (2004): 1678-1688.
2. Fauci SA., et al. "Harrison's Principles of Internal Medicine". Mc Graw Hill Medical (2008).

3. Burnand B., et al. "Serum 25-hydroxyvitamin D: distribution and determinants in Swiss population". The American journal of clinical nutrition 56.3 (1992): 537-540.

4. Wang S. "Epidemiology of vitamin D in health and disease". Nutrition Research Reviews 22.2 (2009): 188-203.

5. Ross AC., et al. "The 2011 report on dietary reference intakes for calcium and vitamin D from the Institute of Medicine: what clinicians need to know". Journal of Clinical Endocrinology and Metabolism 96.1 (2011): 53-58.

6. Holick MF and Chen TC. "Vitamin D deficiency: a worldwide problem with health consequences". The American Journal of Clinical Nutrition 87.4 (2008): 1080-1086.

7. Souberbielle JC., et al. "Prevalence and determinants of vitamin D deficiency in healthy French adults: the VARIETE study". Endocrine 53.2 (2016): 543-550.

8. Abbas S., et al. "Plasma 25-hydroxyvitamin D and premenopausal breast cancer risk in a German case-control study". International Journal of Cancer 124.1 (2009): 250-255.

9. Garland CF., et al. "Vitamin D and prevention of breast cancer: pooled analysis". Journal of Steroid Biochemistry and Molecular Biology 103.3-5 (2007): 708-711.

10. Sørensen IM., et al. "Vitamin D-binding protein and 25-hydroxyvitamin D during pregnancy in mother whose children later developed type 1 diabetes". Diabetes/Metabolism Research and Reviews 32.8 (2016): 883-890.

11. Binkley N., et al. "Laboratory reporting of 25-hydroxyvitamin D results: potential for clinical misinterpretation". Clinical Chemistry 52.11 (2006): 2124-2125.

12. Lissner D., et al. "Stability of vitamin D metabolites in humanblood serum and plasma”. Clinical Chemistry 27.5 (1981): 773774 .

13. Antoniucci DM., et al. "Serum 25-hydroxyvitamin D is unaffected by multiple freeze- thaw cycles". Clinical Chemistry 51.1 (2005): 258-261. 
14. Hollis BW and Wagner CL. "Vitamin D requirements during lactation: high-dose maternal supplementation as therapy to prevent hypovitaminosis D for both the mother and the nursing infant". American Journal of Clinical Nutrition 80.6 (2004): 1752-1758.

15. Horst RL. "The assessment of circulating 25(OH)D and 1,25(OH)2D: where we are and where we are going". Journal of Steroid Biochemistry and Molecular Biology 103.3-5 (2007): 473-476.

16. Holick MF. "Vitamin D status: Measurement, interpretation, and clinical application". Annals of Epidemiology 19.2 (2009): 73-78.

17. International Organization for Standardization (ISO). "International Standard 17511" (2003).

18. Zerwekh JE. "The measurement of vitamin D: analytical aspects". Annals of Clinical Biochemistry 41.4 (2004): 272-281.

19. Horst RL and Hollis BW. "Vitamin D assays and their clinical utility". In: Holick MF, ed. Physiology,

Molecular Biology, and Clinical Applications. Totowa: NJ: Humana Press Inc. (1999): 239-271.

20. Hollis BW. "Editorial: The determination of circulating 25-hydroxyvitamin D: no easy task". The Journal of Clinical Endocrinology and Metabolism 89.7 (2004): 3149-3151.

21. Vogeser M., et al. "Candidate reference method for the quantification of circulating 25-hydroxyvitamin D3 by liquid chromatography-tandem mass spectrometry". Clinical Chemistry 50.8 (2004): 1415-1417.

22. Binkley N., et al. "Assay variation confounds the diagnosis of hypovitaminosis D: a call for standardization". Journal of Clinical Endocrinology and Metabolism 89.7 (2004): 3152-3157.

23. Coldwell RD., et al. "Measurement of 25-hydroxyvitamin D2, 25-hydroxyvitamin D3, 24,25-dihydroxyvitamin D2 and 25,26-dihydroxyvitamin D2 in a single plasma sample by mass fragmentography". Clinica Chimica Acta 180.2 (1989): 157-168.

24. Lensmeyer GL., et al. "HPLC method for 25-hydroxyvitamin D measurement: comparison with contemporary assays". Clinical Chemistry 52.6 (2006): 1120-1126.
25. Terry AH., et al. "Measurement of 25-hydroxyvitamin D by the Nichols ADVANTAGE, DiaSorin LIAISON, DiaSorin RIA, and liquid chromatography-tandem mass spectrometry". Clinical Chemistry 51.8 (2005): 1565-1566.

26. Sahillioğlu B., et al. "Method validation of tandem mass spectrometry for 25-Hydroxyvitamin D3 and comparison of this method with other methods". Turkish Journal of Biochemistry 36.1 (2011): 73-79.

27. Borai A., et al. "Assessment of Becton Dickinson Plain and Serum Separator Tubes in Measurement of 25-Hydroxyvitamin D3 (250HD3) by HPLC and Immunoassay Methods". Journal of clinical laboratory analysis 30.1 (2016): 32-35.

28. Holick MF. "Resurrection of vitamin D deficiency and rickets". Journal of Clinical Investigation 116.8 (2006): 2062-2072.

29. Holick MF. "High prevalence of vitamin D inadequacy and implications for health". Mayo Clinic Proceedings 81.3 (2006): 353-373.

30. Brown EM., et al. "Cloning and characterization of an extracellular Ca2p-sensing receptor from bovine parathyroid". Nature 366.6455 (1993): 575-580.

31. Bouillon R. "Vitamin D: From photosynthesis, metabolism, and action to clinical applications. In: DeGroot LJ, Jameson JL, eds. Endocrinology. Philadelphia: WB Saunders (2001): 10091028.

32. Norman R. Draper and Harry Smith. "Applied Regression Analysis". Wiley (1990).

\section{Volume 3 Issue 8 August 2019}

(C) All rights are reserved by Hülya Çiçek, et al. 